\title{
Interactive approaches for biobjective problems with progressively changing solution sets
}

\author{
Gülşah Karakayaa ${ }^{\mathrm{a}, *}$ and Murat Köksalan ${ }^{\mathrm{b}, \mathrm{c}}$ \\ ${ }^{a}$ Department of Business Administration, Middle East Technical University, 06800 Ankara, Turkey \\ ${ }^{\mathrm{b}}$ Department of Industrial Engineering, Middle East Technical University, 06800 Ankara, Turkey \\ ${ }^{c}$ Operations and Information Management, Georgetown University, Washington, DC 20057, USA \\ E-mail:kgulsah@metu.edu.tr [Karakaya]; koksalan@metu.edu.tr [Köksalan]
}

Received 7 August 2017; received in revised form 19 June 2018; accepted 21 June 2018

\begin{abstract}
In this study, we develop interactive approaches to find a satisfactory alternative of a decision maker (DM) having a quasiconvex preference function where the alternative set changes progressively. In this environment, we keep searching the available set of alternatives and estimating the preference function of the DM. As new alternatives emerge, we make better use of the available preference information and eventually converge to a preferred alternative of the DM. We test our approaches on biobjective, multi-item, multi-round auction problems. The results show that our approaches work well in terms of both the preference function value of the obtained solution and the amount of preference information required.
\end{abstract}

Keywords: multi-objective optimization; progressively changing solution set; interactive approach; multi-attribute auctions

\section{Introduction}

In multi-objective problems, a common assumption is that there is a fixed set of available alternatives from which the decision maker (DM) makes choices. Depending on the nature of the problem, these alternatives may be explicitly available or they may be generated (solving mathematical models) as needed. In practice, the assumption that all alternatives are available at the outset is too strong. In some problems, alternatives may emerge progressively over time. Determination of the winner(s) in a multi-attribute multi-round auction setting is an example for such a situation. In this setting, bidders update their bids at each round creating a new set of alternatives progressively. Korhonen et al. (1993) mention recruiting processes as examples of such environments. E-commerce, where the products sold online, and the real estate market, where the listed houses keep changing continuously.

${ }^{*}$ Corresponding author. 
At any point in time, the available set of alternatives may comprise two subsets: those that are still available from earlier stages and those that have just emerged. Also, even if all the alternatives are available at the outset, the DM may find it cognitively hard to consider all of them at once. After considering a subset of the available alternatives he/she may wish to stop if a satisfactory alternative is reached.

The above discussions are in line with the satisficing and bounded rationality concepts of Herbert Simon (1972), where he argues that there are limits to the "rationality" of humans. The problem's complexity in terms of risks, uncertainty, and the sophistication in the problem environment may be prohibitive for an individual to evaluate everything and come up with the best decision. Simon also argues that only limited, incomplete information may be available about all possible courses of action. His arguments imply that the decision-making environment is typically dynamic, whether due to the nature or the complexity of the problem. Therefore, he argues that an individual's rationality is bounded and that people make satisficing, rather than optimal decisions. He claims that people either reduce their aspirations or search for additional alternatives in case they cannot find satisfactory alternatives. In addition to these difficulties, the dynamic nature of the problem may lead an individual to different sets of alternatives based on the choices made in earlier stages. We will discuss one such case, the biattribute, multi-item, multi-round auction problem, as an example in our computational studies later in this paper.

Korhonen et al. (1993) developed a progressive algorithm that uses pairwise comparisons of the $\mathrm{DM}$ to test the form of his/her underlying preference function and provides a probability range under a set of assumptions for finding better alternatives based on which the DM decides whether or not to continue the selection process. Chun (2015) developed a decision model based on the assumption that the DM has a major attribute to be optimized and minor attributes to be satisfied. He devises search strategies to make a choice under uncertainty.

In this study, we develop algorithms that iterate progressively until finding a satisfactory alternative of a DM. Without loss of generality, we assume that both objectives are of minimization type and the DM's preferences are consistent with a general quasiconvex function that is unknown to us. We keep iterating and progressively evaluating alternatives as they become available until a suitable stopping condition is satisfied. Quasiconcave preference functions have been considered to represent human behavior well for maximization-type objectives (see Köksalan et al., 1984; Silberberg and Suen, 2001, pp. 260-261) and have been used in the literature extensively (see, e.g., Korhonen et al., 1984; Lokman et al., 2016; Özpeynirci et al., 2017). When the objectives are of minimization type, quasiconvex preference functions are suitable in an identical fashion. There are approaches that test whether a DM's preferences are consistent with such functions (Korhonen et al., 1986; Köksalan and Sagala, 1995) and they can be used as preprocessors to see if the assumption is approximately satisfied.

Karakaya and Köksalan (2016) consider the multi-round auction problem under the assumption of a linear preference function. In this paper, we consider a generalized version of their problem from several perspectives. Our approach is not specific to auctions and can be applied to any problem that has a progressively changing solution set. We address issues such as when to stop with a satisfactory solution in an environment where new solutions keep emerging in time.

Another general aspect of this paper is that, it allows for a wider class of preference structures (quasiconvex preference functions) that have been considered to represent human behavior well. We develop models and an algorithm to find the most preferred solution of a DM. We introduce 
several variations of the algorithm trading off the solution quality with the computational effort in a controlled manner. We demonstrate the performances of different versions of the algorithm with extensive computational experiments.

In Section 2, we introduce relevant definitions and develop the approaches. We provide an example application area and demonstrate the performance of the approaches in Section 3. We present our concluding remarks in Section 4.

\section{The approach}

In this section, we provide some definitions, develop an approach and its variations.

\subsection{Definitions}

We assume that new sets of solutions emerge at certain periods that we refer to as rounds. The definitions and the solution sets refer to the round under consideration. We omit symbols representing rounds for the sake of simplicity of the notation. We define the general multi-objective optimization problem (in a given round) as follows:

$$
\begin{aligned}
& \text { "Min" }\left(z_{1}(\boldsymbol{x}), \ldots, z_{J}(\boldsymbol{x})\right)^{T} \\
& \text { s.t. } \quad \boldsymbol{x} \in \boldsymbol{X},
\end{aligned}
$$

where $z_{j}(\boldsymbol{x})$ is the $j$ th objective function, $\boldsymbol{x}$ is the decision vector, and $\boldsymbol{X}$ is the feasible decision set. At this point, we do not make any assumptions about the forms of $z_{j}$ s. We assume that $\boldsymbol{X}$ is a bounded set. We use the quotation marks to emphasize that the minimization of a vector is not a well-defined mathematical operation.

Let $\boldsymbol{Z}$ be the image of $\boldsymbol{X}$ in the objective space. An objective vector $\boldsymbol{z}^{i}=\left(z_{1}^{i}, \ldots, z_{J}^{i}\right)^{T} \in \boldsymbol{Z}$ is said to dominate $\boldsymbol{z}^{k}=\left(z_{1}^{k}, \ldots, z_{J}^{k}\right)^{T} \in \boldsymbol{Z}$ if and only if $z_{j}^{i} \leq z_{j}^{k}$ for all $j$ and $z_{j}^{i}<z_{j}^{k}$ for at least one $j$. If there does not exist any $z^{i} \in Z$ that dominates $z^{k}$, then $z^{k}$ is said to be nondominated.

Let $\boldsymbol{z}^{i} \in \boldsymbol{Z}, i=1, \ldots, n$ be distinct solutions. Let $\boldsymbol{Y}$ be the set of all convex combinations of $\boldsymbol{z}^{i}$, where $\boldsymbol{Y}=\left\{\boldsymbol{y}: \boldsymbol{y}=\sum_{i=1}^{n} \mu_{i} \boldsymbol{z}^{i}, \sum_{i=1}^{n} \mu_{i}=1, \mu_{i} \geq 0\right\}$.

A nondominated solution, $\boldsymbol{z}^{v}$, is said to be unsupported nondominated if and only if there exists $\boldsymbol{y} \in \boldsymbol{Y}$ such that $y_{j} \leq z_{j}^{v}$ for all $j$ and $y_{j}<z_{j}^{v}$ for at least one $j$. A nondominated solution, $\boldsymbol{z}^{v}$, is said to be nonextreme-supported nondominated if and only if there exists $\boldsymbol{y} \in \boldsymbol{Y}$ such that $y_{j}=z_{j}^{v}$ for all $j$ and the coefficient of $\boldsymbol{z}^{v}$ in $\boldsymbol{Y}$ is zero (i.e., $\mu_{v}=0$ ). A nondominated solution, $\boldsymbol{z}^{v}$, is said to be extreme-supported nondominated if and only if there does not exist $\boldsymbol{y} \in \boldsymbol{Y}$ such that $y_{j} \leq z_{j}^{v}$ for all $j$ and $\mu_{v}=0$.

We demonstrate the classification of the solutions based on the domination rules where both objectives are to be minimized in Fig. 1.

Let $z^{s}, z^{r} \in Z$, and $z^{r}$ be extreme-supported nondominated. $z^{s}$ is said to be adjacent nondominated to $z^{r}$ if and only if their convex combinations are not dominated by the convex combinations of solutions in $\boldsymbol{Z}-\left\{\boldsymbol{z}^{s}\right\}$. In biobjective problems, an extreme-supported nondominated solution can have at most two distinct adjacent nondominated solutions (see Ramesh et al., 1990). We label these adjacent nondominated solutions as east and west where the east adjacent nondominated solution 


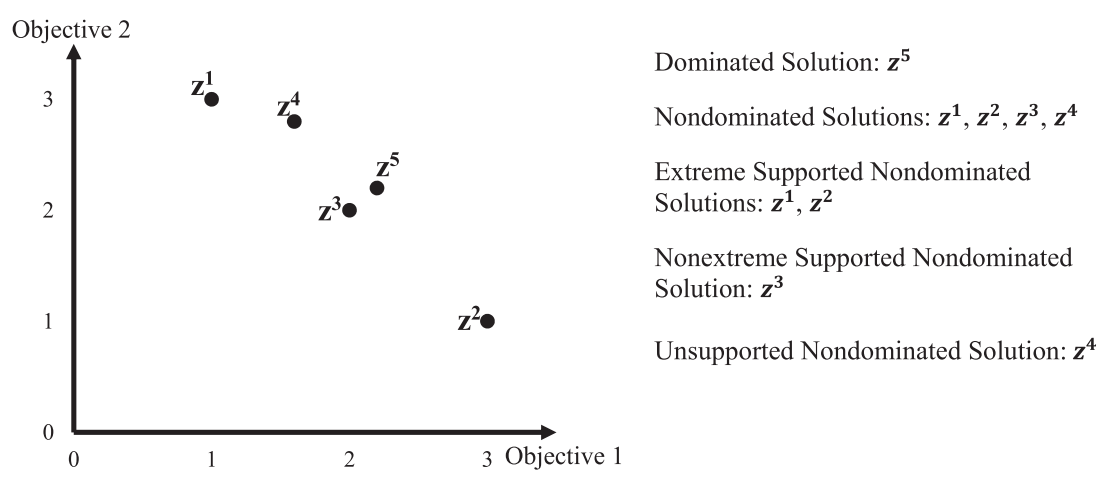

Fig. 1. Classification of the solutions where $z^{1}=(\mathbf{1}, \mathbf{3})^{T}, z^{2}=(\mathbf{3}, \mathbf{1})^{T}, z^{3}=(\mathbf{2}, \mathbf{2})^{T}=\mathbf{0 . 5} z^{1}+\mathbf{0 . 5} z^{2}$, $z^{4}=(1.6,2.8)^{T}$, and $z^{5}=(2.2,2.2)^{T}$.

has a higher value than the reference solution in objective 1, whereas the west adjacent nondominated solution has a higher value than the reference solution in objective 2 .

Let $u: R^{J} \rightarrow R^{1}$ be a quasiconvex function. By definition of a quasiconvex function, $u\left(\sum_{i=1}^{n} \mu_{i} z^{i}\right) \leq \max _{i}\left\{u\left(z^{i}\right)\right\}$ for $\sum_{i=1}^{n} \mu_{i}=1, \mu_{i} \geq 0$, and $z^{i} \in \boldsymbol{Z}, i=1, \ldots, n$. We rewrite Lemma 2 of Korhonen et al. (1984) developed for a quasiconcave preference function to be maximized for the case of a quasiconvex function to be minimized.

Lemma 1. Let $u: R^{J} \rightarrow R^{1}$ be a nondecreasing quasiconvex preference function and consider distinct solutions $z^{i} \in R^{J}, i=1, \ldots, n$. Let $u\left(z^{i}\right)<u\left(z^{k}\right), i \neq k$. Let $\quad \boldsymbol{C}\left(z^{i}, z^{k}: i=1, \ldots, n, i \neq k\right)=$ $\left\{\boldsymbol{z}: \boldsymbol{z}=\boldsymbol{z}^{k}+\sum_{i=1, i \neq k}^{n} \mu_{i}\left(\boldsymbol{z}^{k}-\boldsymbol{z}^{i}\right), \mu_{i} \geq 0\right\}$. Then, $u(\boldsymbol{z}) \geq u\left(\boldsymbol{z}^{k}\right)$.

The proof of the lemma directly follows from that of Korhonen et al. (1984).

Considering that $u$ represents a DM's preference function where smaller values of $u$ are more preferable, the lemma implies that the solutions in cone $C\left(z^{i}, z^{k}: i=1, \ldots, n, i \neq k\right)$ or dominated by any point in that cone are at most as preferred as $z^{k}$ and inferior to $z^{i}, i=1, \ldots, n, i \neq k$. Throughout this paper, we assume both objectives are of minimization type, without loss of generality and will refer to the points that are in or dominated by a cone as a cone-dominated point. We use the notation $C\left(z^{i}, z^{k}\right)$ to depict two-point cones where $z^{i}$ is preferred to $z^{k}\left(\right.$ denoted $\left.z^{i} \succ z^{k}\right)$. We use the terms solution and alternative interchangeably. For the sake of simplicity, we will drop the term "nondominated" and refer to supported nondominated, extreme-supported nondominated, and adjacent nondominated solutions as supported, extreme supported, and adjacent solutions, respectively, in the rest of the paper.

\subsection{The interactive algorithm ( $Q C X-u)$}

We develop an interactive algorithm, $Q C X$ - $u$, to find a satisfactory solution of a DM whose preferences are consistent with a quasiconvex preference function for the biobjective problem, where the solution sets change progressively. In $Q C X$ - $u$, we keep evaluating alternatives as they become available and the algorithm iterates until a termination condition is satisfied.

In $Q C X-u$, in a given round, we first find the most preferred supported solution among the available solutions in that round. Then, we continue the search with unsupported solutions to find 


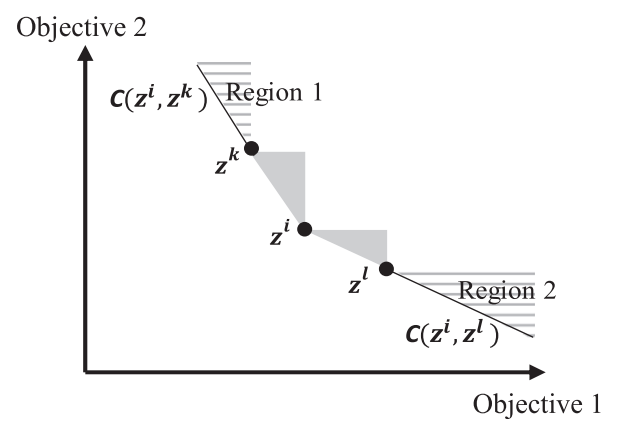

Fig. 2. Reduced search space.

the most preferred solution of that round. We move to the next round with the best solution of the current round appended to the updated solutions for the upcoming round. We also carry the past preference information and implied restrictions on the search region to the next round. We continue considering new solutions progressively in each round until a suitable stopping condition is satisfied.

An important aspect of $Q C X$ - $u$ is to utilize the properties of a quasiconvex function together with the available preference information to reduce the search space. Assume that the DM's preferences are consistent with a quasiconvex function of the two objectives. Let $\boldsymbol{z}^{i}$ be preferred to both its adjacent solutions $z^{k}$ and $z^{l}$. Due to Lemma 1 , all points in Region 1 are cone-dominated by cone $\boldsymbol{C}\left(\boldsymbol{z}^{i}, \boldsymbol{z}^{k}\right)$ and all points in Region 2 are cone-dominated by cone $\boldsymbol{C}\left(\boldsymbol{z}^{i}, \boldsymbol{z}^{l}\right)$ in Fig. 2. Eliminating the cone-inferior regions together with the dominated regions, we are left with the two shaded triangular regions. The most preferred solution of the corresponding round is either $z^{i}$ or is a solution located in the shaded triangular regions. The fact that we have only two objectives always reduces the final search region into at most two triangular regions formed by a supported solution and its less preferred adjacent solutions.

It is well known that under a linear preference function, considering all available set of solutions, a solution that is preferred to all its adjacent solutions is the most preferred solution. There exist algorithms that find the most preferred supported solution in such environments. Zionts (1981) and Karakaya and Köksalan (2016) are two such approaches in different contexts. Under a quasiconvex preference function, we discussed above that the most preferred solution of the current round appears in the triangles formed by the most preferred supported solution and its adjacent solutions in the corresponding round.

We first discuss the details of different aspects of $Q C X-u$ and then give its steps.

In $Q C X$ - $u$, we first find the most preferred supported solution of a given round. We then continue our search with the unsupported solutions in the admissible search region to find the most preferred solution of that round. Our solution set is updated in each round based on the changes in the problem environment. We refer to the current best solution as the incumbent. In search for an incumbent solution preferred to its adjacent solutions, we keep updating the incumbent solving a model whenever an adjacent is preferred to the current incumbent. In finding a new (candidate) incumbent, we first solve a model that estimates the weights of a linear preference function that is most consistent with the past preferences of the DM. We construct cones implied by a quasiconvex preference function and past preferences of the DM in order to eliminate the solutions that are 
inferior with respect to these cones. Hence, we keep finding the best solution of each round and end up with a satisfactory/overall best solution at termination.

Let $z^{*}$ be the current best solution (incumbent), $z^{P R E V}$ be the best solution of the previous round, $\boldsymbol{Z}$ be the available set of solutions, and $\boldsymbol{P}=\left\{\left(z^{i}, z^{k}\right): z^{i} \succ z^{k}\right\}$ be the set of responses on pairs of solutions showing the past preferences of the DM. Let $\hat{\boldsymbol{w}}=(\hat{w}, 1-\hat{w})$, where $\hat{w}$ be the estimated weight of objective 1 and $\rho$ be a small positive constant.

We next discuss the weight estimation and candidate incumbent search procedures.

\section{Estimating weights}

We solve the $\left(W_{\text {est }}\right)$ model to find a feasible weight vector satisfying the preference constraints constructed based on the preference information obtained from the DM.

$$
\begin{aligned}
& \operatorname{Model}\left(W_{e s t}\right) \\
& \operatorname{Max} \varepsilon \\
& \text { s.t. } \\
& \hat{w} \cdot z_{1}^{i}+(1-\hat{w}) \cdot z_{2}^{i} \leq \hat{w} \cdot z_{1}^{k}+(1-\hat{w}) \cdot z_{2}^{k}-\rho-\varepsilon \quad \text { for each }\left(z^{i}, z^{k}\right) \in \boldsymbol{P}, \\
& \varepsilon \leq \hat{w} \leq 1-\varepsilon, \\
& \varepsilon \geq 0 .
\end{aligned}
$$

The objective is to find the maximum $\varepsilon$ value satisfying the constraints. That is, the minimum deviation from each constraint is maximized in the spirit of Köksalan et al. (1984) to find central weights. The preferences of the DM are modeled by (2). We normalize the weights by (3) and enforce $\varepsilon$ to be nonnegative by (4). Note that constraints (2)-(4) essentially imply upper and lower bounds for $\hat{w}$, which can easily be calculated. The solution to this model corresponds to the midpoint of this range.

We assume that the DM's preferences are consistent with a quasiconvex preference function and the above model locally approximates it with a linear function in (2). If the solution set is not empty, then we use the weights found by the model in our algorithm. Otherwise, we relax the constraints causing infeasibility and solve the following linear model, Model $\left(W_{\text {inf }}\right)$, to find a feasible set of weights that are most consistent with the past preferences of the DM. There are alternative ways of handling infeasibility such as minimizing the number of constraints that are allowed to be violated or removing constraints corresponding to the oldest responses of the DM (see Köksalan et al., 1984; Chinneck, 2008).

$$
\begin{aligned}
& \operatorname{Model}\left(W_{\text {inf }}\right) \\
& \operatorname{Min} v+\theta \cdot \sum_{\left(z^{i}, z^{k}\right) \in \boldsymbol{P}} \varepsilon_{i, k} \\
& \text { s.t. } \\
& \hat{w} \cdot z_{1}^{i}+(1-\hat{w}) \cdot z_{2}^{i} \leq \hat{w} \cdot z_{1}^{k}+(1-\hat{w}) \cdot z_{2}^{k}-\rho+\varepsilon_{i, k} \quad \text { for each }\left(\boldsymbol{z}^{i}, \boldsymbol{z}^{k}\right) \in \boldsymbol{P}, \\
& v \geq \varepsilon_{i, k} \quad \text { for each }\left(\boldsymbol{z}^{i}, \boldsymbol{z}^{k}\right) \in \boldsymbol{P},
\end{aligned}
$$




$$
\begin{aligned}
& \varepsilon_{i, k} \geq 0 \quad \text { for each }\left(z^{i}, z^{k}\right) \in \boldsymbol{P}, \\
& \hat{w} \geq 0,
\end{aligned}
$$

where $v$ is introduced to capture the maximum amount of infeasibility and $\theta$ is a small positive constant. For each preference constraint, we add new variables $\varepsilon_{i, k}$ s to represent the amount of violation of the corresponding constraint. The positive $\varepsilon_{i, k}$ values indicate that the corresponding constraints are violated. In the objective function of $\left(W_{i n f}\right)$, we introduce an augmentation $\left(\theta \cdot \sum_{\left(z^{i}, z^{k}\right) \in \boldsymbol{P}} \varepsilon_{i, k}\right)$ as a secondary objective to break ties when the minimum $v$ value is not unique. By construction, the $\left(W_{\text {inf }}\right)$ model is always feasible and we use the estimated weight vector, $\hat{\boldsymbol{w}}=(\hat{w}, 1-\hat{w})$, found by solving $\left(W_{\text {inf }}\right)$.

\section{Finding a new incumbent}

When an adjacent solution is preferred to the current incumbent, the incumbent is replaced with the preferred adjacent solution. Then, a new candidate is searched to be compared with the incumbent. For this aim, we solve Model (NewSol) using the estimated weight vector, $\hat{w}$, most consistent with the past preferences of the DM.

$$
\begin{aligned}
& \text { Model (NewSol) } \\
& \operatorname{Min} \hat{w} \cdot z_{1}+(1-\hat{w}) \cdot z_{2} \\
& \text { s.t. } \\
& \hat{w} \cdot z_{1}+(1-\hat{w}) \cdot z_{2} \leq \hat{w} \cdot z_{1}^{*}+(1-\hat{w}) \cdot z_{2}^{*}-\rho \\
& z_{2} \leq z_{2}^{k}-\rho+M \cdot t\left(z^{i}, z^{k}\right) \quad \text { for each }\left(z^{i}, z^{k}\right) \in \boldsymbol{P}, z_{1}^{i}>z_{1}^{k} \text {, } \\
& w\left(z^{i}, z^{k}\right) \cdot z_{1}+\left(1-w\left(z^{i}, z^{k}\right)\right) \cdot z_{2} \leq w\left(z^{i}, z^{k}\right) \cdot z_{1}^{k}+\left(1-w\left(z^{i}, z^{k}\right)\right) \cdot z_{2}^{k}-\rho \\
& +M \cdot\left(1-t\left(z^{i}, z^{k}\right)\right) \quad \text { for each }\left(z^{i}, z^{k}\right) \in \boldsymbol{P}, z_{1}^{i}>z_{1}^{k}, \\
& z_{1} \leq z_{1}^{k}-\rho+M \cdot t\left(z^{i}, z^{k}\right) \quad \text { for each }\left(z^{i}, z^{k}\right) \in \boldsymbol{P}, z_{1}^{i}<z_{1}^{k} \text {, } \\
& w\left(\boldsymbol{z}^{i}, \boldsymbol{z}^{k}\right) \cdot z_{1}+\left(1-w\left(\boldsymbol{z}^{i}, \boldsymbol{z}^{k}\right)\right) \cdot z_{2} \leq w\left(\boldsymbol{z}^{i}, \boldsymbol{z}^{k}\right) \cdot z_{1}^{k}+\left(1-w\left(\boldsymbol{z}^{i}, \boldsymbol{z}^{k}\right)\right) \cdot z_{2}^{k}-\rho \\
& +M \cdot\left(1-t\left(z^{i}, z^{k}\right)\right) \quad \text { for each }\left(z^{i}, z^{k}\right) \in \boldsymbol{P}, z_{1}^{i}<z_{1}^{k}, \\
& z \in Z, \quad t\left(z^{i}, z^{k}\right) \in\{0,1\}, \forall\left(z^{i}, z^{k}\right) \in \boldsymbol{P},
\end{aligned}
$$

where $\rho$ is a small positive constant, $M$ is a sufficiently large positive number, $t\left(z^{i}, z^{k}\right)$ is a binary variable for each pairwise preference of the DM in set $\boldsymbol{P}$, and $w\left(\boldsymbol{z}^{i}, \boldsymbol{z}^{\boldsymbol{k}}\right)=\frac{z_{2}^{i}-z_{2}^{k}}{z_{1}^{k}-z_{2}^{k}-z_{1}^{i}+z_{2}^{i}}$ (i.e., $w\left(\boldsymbol{z}^{i}, \boldsymbol{z}^{\boldsymbol{k}}\right)$ and $\left(1-w\left(\boldsymbol{z}^{i}, \boldsymbol{z}^{\boldsymbol{k}}\right)\right)$ are the weights of the linear function passing through solutions $\boldsymbol{z}^{i}$ and $\left.\boldsymbol{z}^{\boldsymbol{k}}\right)$.

In $(\mathrm{NewSol})$, the objective (10) is to find the solution that minimizes the weighted linear function using $\hat{\boldsymbol{w}}$. Constraint (11) guarantees that the estimated linear preference function value of the new solution is less than that of the incumbent. Remaining constraints are the cone constraints implied by Lemma 1 and are used to avoid inferior solutions based on a quasiconvex preference function and past preferences of the DM (also see Fig. 2). Constraints (12) and (13) are enforced to avoid the set of cone-dominated points (Region 1) in Fig. 2. The complement of Region 1 characterizes 
the admissible region that is nonconvex. We use binary variables to represent this region. Similarly, Constraints (14) and (15) are enforced to avoid the set of cone-dominated points (Region 2) in Fig. 2.

Karakaya and Köksalan (2016) developed an exact interactive algorithm for biattribute, multiitem, multi-round auctions assuming that the DM has a linear preference function. The multiround auction problem is a special case of an environment where the solution set changes progressively. Furthermore, the linear preference function simplifies the solution process. Our $Q C X-u$ algorithm accounts for the changing environment and considers a quasiconvex preference function, hence generalizing the problem in both respects making it applicable to a wide variety of situations.

We utilize some tools developed by Karakaya and Köksalan (2016). We modify their approach of finding adjacent solutions to an incumbent by introducing bounds to eliminate the inferior regions in the solution space. To find the weights of an estimated linear preference function that are most consistent with the DM's past preferences, we use their model (IR) without allowing for indifference preferences. Finally, at the beginning of each round, after finding a new incumbent we apply the Status-of-the-Solution $(S o S)$ procedure (see Karakaya and Köksalan, 2016 for details) to determine whether the best solution found so far, $\boldsymbol{z}^{\boldsymbol{P R} \boldsymbol{E} \boldsymbol{V}}$, is extreme supported or not.

The steps of $Q C X-u$

Let $\boldsymbol{z}^{a d j}$ be a nondominated solution adjacent to the incumbent, and $\boldsymbol{z}^{E}$ and $\boldsymbol{z}^{W}$ be the east and west adjacent solutions to the incumbent, respectively. Select a direction DIR = east (without loss of generality) to search for the nondominated solution adjacent to the incumbent where $D I R=$ east (west) corresponds to searching for east (west) adjacent solution. Let $r n d=n e w$ indicate the beginning of a new round, $h$ be the iteration counter and set $h=0$. Set $r n d=$ old to indicate that the current round is in progress. Recall the notation $z^{*}, z^{P R E V}, \boldsymbol{Z}, \boldsymbol{P}=\left\{\left(\boldsymbol{z}^{i}, \boldsymbol{z}^{k}\right): \boldsymbol{z}^{i} \succ \boldsymbol{z}^{k}\right\}$, $\hat{\boldsymbol{w}}=(\hat{w}, 1-\hat{w})$, and $\rho$ defined above.

Step 0: Select an arbitrary set of weights, find the incumbent solution, $z^{*}$, that minimizes the resulting weighted linear function. Go to Step 2.

Step 1: Set $h \leftarrow h+1$. Solve $(N e w S o l)$ to find a new candidate for incumbent, $z^{h}$. If the model is infeasible, then there exists no $z^{h}$, go to Step 2; otherwise, go to Step 1.1.

Step 1.1: If $r n d=n e w$, check the status of $z^{P R E V}$. If $z^{P R E V}$ is extreme supported, go to Step 1.3; otherwise set $z^{*}=z^{h}$ and go to Step 2 .

Step 1.2: If $r n d=$ old, go to Step 1.3.

Step 1.3: If $z_{1}^{h}<z_{1}^{*}$, set $D I R=$ west; otherwise set $D I R=$ east. Ask the DM $\boldsymbol{z}^{*}$ versus $\boldsymbol{z}^{\boldsymbol{h}}$. If

- $\boldsymbol{z}^{\boldsymbol{h}}$ is preferred to $\boldsymbol{z}^{*}$, let $\boldsymbol{P} \leftarrow \boldsymbol{P} \cup\left(\boldsymbol{z}^{h}, \boldsymbol{z}^{*}\right)$. Set $\boldsymbol{z}^{*}=\boldsymbol{z}^{\boldsymbol{h}}$ and go to Step 2 .

- $\boldsymbol{z}^{*}$ is preferred to $\boldsymbol{z}^{h}$, let $\boldsymbol{P} \leftarrow \boldsymbol{P} \cup\left(\boldsymbol{z}^{*}, \boldsymbol{z}^{h}\right)$. Switch the value of $D I R$ and go to Step 2 .

Step 2: If $D I R=$ east, go to Step 2.1, otherwise go to Step 2.2.

Step 2.1: If there does not exist $z^{E}$, go to Step 2.3. Otherwise, set $z^{a d j}=z^{E}$ and go to Step 2.4. Step 2.2: If there does not exist $z^{W}$, go to Step 2.3. Otherwise, set $z^{a d j}=z^{W}$ and go to Step 2.4. Step 2.3: If both $z^{E}$ and $z^{W}$ have been evaluated, go to Step 4. Otherwise, switch the value of $D I R$ and go to Step 2. 
Step 2.4: Ask the DM $z^{*}$ versus $z^{a d j}$. If

- $\boldsymbol{z}^{a d j}$ is preferred to $z^{*}$, let $\boldsymbol{P} \leftarrow \boldsymbol{P} \cup\left(\boldsymbol{z}^{a d j}, \boldsymbol{z}^{*}\right)$. Set $\boldsymbol{z}^{*}=\boldsymbol{z}^{\text {adj }}$, rnd $=$ old, and go to Step 3 . - $\boldsymbol{z}^{*}$ is preferred to $\boldsymbol{z}^{a d j}$, let $\boldsymbol{P} \leftarrow \boldsymbol{P} \cup\left(\boldsymbol{z}^{*}, \boldsymbol{z}^{a d j}\right)$ and go to Step 2.3.

Step 3: Find a feasible set of weights that are most consistent with the past preferences of the DM. Go to Step 1.

Step 4: If $D I R=$ east, go to Step 4.1, otherwise go to Step 4.2.

Step 4.1: If there does not exist $z^{E}$, go to Step 4.3. Otherwise, set $z^{a d j}=z^{E}$ and go to Step 4.4. Step 4.2: If there does not exist $z^{W}$, go to Step 4.3. Otherwise, set $z^{a d j}=z^{W}$ and go to Step 4.4. Step 4.3: If both directions have been searched and no adjacent solution has been found, set $r n d=n e w$ and go to Step 5. Otherwise, switch the value of $D I R$ and go to Step 4.

Step 4.4: Ask the DM $z^{*}$ versus $z^{\text {adj }}$. If

- $\boldsymbol{z}^{a d j}$ is preferred to $z^{*}$, let $\boldsymbol{P} \leftarrow \boldsymbol{P} \cup\left(z^{a d j}, z^{*}\right)$. Set $z^{*}=\boldsymbol{z}^{a d j}$ and go to Step 4 .

- $z^{*}$ is preferred to $z^{a d j}$, let $\boldsymbol{P} \leftarrow \boldsymbol{P} \cup\left(z^{*}, z^{a d j}\right)$. Switch the value of $D I R$ and go to Step 4 .

Step 5: If the termination condition is satisfied, go to Step 6; otherwise, set $z^{P R E V}=z^{*}$, update $\boldsymbol{Z}$, and go to Step 3.

Step 6: Stop, $z^{*}$ is a satisfactory solution, that is, the most preferred solution among the existing solutions.

In $Q C X-u$, we keep an incumbent solution throughout. In Step 1, we search for a new candidate for the incumbent. At the start of each new round we replace the incumbent with the new candidate if it satisfies the required conditions. We then find solutions adjacent to the incumbent and ask the DM his/her preferences between the incumbent and its adjacent solutions in Step 2. In case the incumbent is preferred to all its adjacent solutions, we conclude that it is the most preferred supported solution among the existing solutions and continue the search with unsupported solutions. Whenever an adjacent solution is preferred to the incumbent, we update estimated weights in Step 3 considering all past preferences of the DM. In Step 4, we search for adjacent solutions to the incumbent in the reduced region. We reach Step 5 once we find the most preferred solution of the current round and stop if the termination condition is satisfied. Otherwise, after updating the set of solutions for the new round, the algorithm continues with Step 1.

In the above algorithm, we fully search the reduced region in each round (er). There are alternative ways of searching the reduced region. We refer to the original version as $Q C X-u(e r)$ and discuss three variations next.

\section{Variations of $Q C X-u$}

$Q C X-u(e r)$ guarantees finding the most preferred solution of the rounds until termination among the available solutions by searching the whole reduced (triangular) regions. In many applications, one would expect some highly preferred solutions to emerge at later rounds. Under such circumstances, it may not be necessary to search the whole solution space in earlier rounds. As more preference information is gathered, it would be possible to exclude larger portions of the solution space that contain less preferred solutions. These observations lead us to develop variations that search the solution space partially in earlier rounds and make a more thorough search in later rounds. We develop three variations that (a) search the triangular regions only in the last round, (b) search different-size bands of solutions in the triangular regions in each round, and (c) enforce 


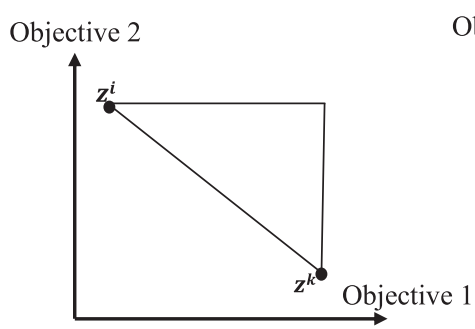

(a) The starting triangular region of round $r$

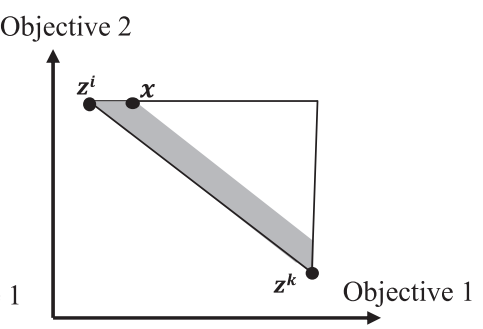

(b) The band used in round $r$

Fig. 3. An example reduced region in $Q C X-u$ (band).

a progressively increasing maximum number of questions $(m n q)$ bound in each round. We denote these algorithms as $Q C X-u(l r), Q C X-u$ (band), and $Q C X-u(m n q)$, respectively.

$Q C X-u(l r)$

In this version of the algorithm, we simplify Step 4 and avoid searching inside the triangular regions until the last round. We only check whether $z^{P R E V}$ is in the triangular regions in the intermediate rounds. If so, we ask the DM to compare the incumbent with $z^{P R E V}$ and make sure to keep the best solution found so far between rounds. In the final round, as in $Q C X-u(e r)$, we search the whole triangular regions to guarantee finding the most preferred one of the available solutions at that round.

\section{$Q C X-u$ (band)}

In this version, we search a portion of the reduced region in Step 4 in the intermediate rounds, again with the intention of reducing the required preference information.

In early rounds we search small portions of the triangular regions and keep increasing the searched proportion of the triangular region of each successive round. We expect that the solutions emerge in later rounds to include some that have better preference function values than the incumbent. By increasing the proportions of searched regions successively, we increase the amount of required preference information gradually without sacrificing much from the quality of the solution obtained at termination. In the final round of the process, we search the whole triangular regions as in the previous versions. We next explain the specifics.

We define a band to be searched in each round. This band has a certain proportion of the area of the triangular region of the current round. The proportion depends on the current round number, $r$, and the round number, $R$, at which we wish to cover the whole triangle. More specifically, if $A(r)$ is the area of the triangle at round $r$, then we search a band having an area of $\min \left\{1, \frac{r}{R}\right\} \cdot A(r)$ in round $r$. That is, if $r<R$, we search a portion of the area $A(r)$, whereas we search the whole area $A(r)$ if $r \geq R$. We use the similarity of triangles in defining the specific band to be searched. To illustrate, suppose that at the beginning of Step 4 of round $r$, we have the triangular region in Fig. 3a.

We define the band as shown in Fig. 3b. We calculate point $\boldsymbol{x}=\left(x_{1}, x_{2}\right)^{T}$ to bound the search region where $x_{2}=z_{2}^{i}$ and using similarity $x_{1}=z_{1}^{k}-\sqrt{\max \left\{0, \frac{R-r}{R}\right\}} \cdot\left(z_{1}^{k}-z_{1}^{i}\right)$. To find adjacent 
solutions that lie in the band in Step 4, we apply a procedure similar to that in $Q C X$-u (er) adding the following constraint to bound the search region.

$$
w\left(\boldsymbol{z}^{i}, \boldsymbol{z}^{k}\right) \cdot z_{1}+\left(1-w\left(\boldsymbol{z}^{i}, \boldsymbol{z}^{k}\right)\right) \cdot z_{2} \leq w\left(\boldsymbol{z}^{i}, \boldsymbol{z}^{k}\right) \cdot x_{1}+\left(1-w\left(\boldsymbol{z}^{i}, \boldsymbol{z}^{k}\right)\right) \cdot x_{2},
$$

where

$$
w\left(z^{i}, z^{k}\right)=\frac{z_{2}^{i}-z_{2}^{k}}{z_{1}^{k}-z_{2}^{k}-z_{1}^{i}+z_{2}^{i}} .
$$

We should note that to keep the best solution between rounds, we ask the DM to compare the incumbent with $z^{P R E V}$ even if $z^{P R E V}$ does not lie in the band, so long as $z^{P R E V}$ is not in a cone inferior region. We need not ask the DM in the latter case as we know that $z^{P R E V}$ is inferior.

\section{$Q C X-u(m n q)$}

In $Q C X-u$ (band), if there are many unsupported solutions in the band of the current round, the amount of preference information asked of the DM may be large. In order to keep the preference information low, we may limit the number of unsupported solutions found in each round. We develop $Q C X-u(m n q)$ for this purpose.

Rather than increasing the search area as in $Q C X-u$ (band), we keep increasing the maximum number of solutions searched in the triangular regions in successive rounds. Let $M N Q$ be the predetermined maximum number of solutions searched in the triangular regions of round 1 . In round $r$, we limit the number of solutions searched to at most $r \cdot M N Q$ in the triangular regions of round $r$.

In order to keep the best solution evaluated by the DM throughout the algorithm, we ask him/her to compare the incumbent with $\boldsymbol{z}^{P R E V}$ when necessary, as above.

In all our algorithms, we assume that the DM can choose the preferred one of a pair of solutions. One might think of situations where the DM is indifferent between some solutions or even is somewhat inconsistent in some of his/her preferences. Our algorithms can easily be extended to handle such cases utilizing the approaches developed in Karakaya and Köksalan (2016).

\section{Computational results on auction problems}

To test the performance of the algorithm, we use the settings of the biattribute multi-round auction problem in Karakaya and Köksalan (2016). Specifically, we consider a combinatorial reverse auction where bidders are the sellers and the auctioneer (i.e., the DM) is the buyer. We use two attributes, price and defect rate, where smaller values are preferred in both attributes by the buyer. Each seller is assumed to have a convex cost function based on which the sellers update their bids as the rounds progress. In Karakaya and Köksalan (2016), a decision support tool that aids the sellers to update their bids is developed. It estimates the underlying preference function of the DM based on his/her past preferences. Utilizing this estimation, it provides information to sellers for the bid update process. Sellers update their bids accordingly, new bid combinations are found, and the auction continues until a suitable stopping condition is satisfied. To demonstrate the performance of our algorithms, we assume without loss of generality that the new solutions (bid combinations) are somehow obtained without going into the details of the bid-generation process for the sake of 


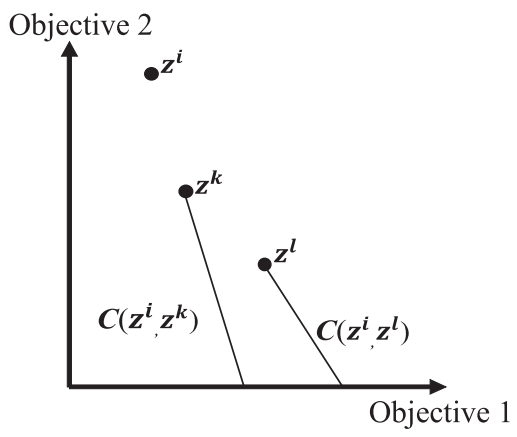

Fig. 4. An example for redundant cones.

simplicity. For the specifics of the problem setting and the generation of new bids, the reader is referred to Karakaya and Köksalan (2016). We should note that, bids are updated based on the preference information gathered from the DM which is directly related with the questions asked to the DM. Since the questions asked to the DM may differ, the emerging bids may differ as well depending on the version of the algorithm.

As discussed earlier, we use cone constraints to avoid inferior solutions based on a quasiconvex preference function and past preferences of the DM. We introduce a binary variable in each cone constraint pair in order to characterize a cone. To improve computational efficiency, we identify and eliminate redundant cones, and reduce the required binary variables. Ramesh et al. (1988) solve a linear programming model to check the redundancy of two-point cones and Lokman et al. (2016) develop a procedure to detect redundant cones without solving a model. We apply the latter approach to eliminate redundant cones.

To illustrate, consider the solutions in Fig. 4 and assume that the DM prefers $z^{i}$ to both $z^{k}$ and $z^{l}$. Based on the preferences of the DM, we generate two cones, $C\left(z^{i}, z^{k}\right)$ and $\boldsymbol{C}\left(z^{i}, \boldsymbol{z}^{l}\right)$. However, since the inferior region implied by $\boldsymbol{C}\left(\boldsymbol{z}^{i}, \boldsymbol{z}^{l}\right)$ is a subset of that of $\boldsymbol{C}\left(\boldsymbol{z}^{i}, \boldsymbol{z}^{\boldsymbol{k}}\right)$, the former is a redundant cone and can be eliminated.

We test the performance of the algorithm for different versions considering the number of questions asked to the DM. For each version, we check the percentage deviations of the preference function values of the solutions found by $Q C X$ - $u$ from an optimal solution. Karakaya and Köksalan (2016) find the overall optimal solution under full information case (about the buyer's preference function) without allowing information sharing between sellers about their cost functions. They denote the resulting solution as the Decentralized solution. More specifically, let $z^{Q C X u}$ be the final solution found by $Q C X-u$. We calculate the percentage deviation of the solution found for any problem instance and for any variation of $Q C X$ - $u$ as follows:

$$
\left(\Delta z^{Q C X-u}\right)=\frac{u\left(z^{Q C X-u}\right)-u(\text { Decentralized })}{u(\text { Decentralized })} \cdot 100 .
$$

We simulate the preferences of the buyer with three quasiconvex underlying preference functions: (a) weighted linear $\left(\min _{z^{i} \in \boldsymbol{Z}} w z_{1}^{i}+(1-w) z_{2}^{i}\right)$, (b) weighted Euclidean $\left(\min _{z^{i} \in \boldsymbol{Z}}\left(\left(w z_{1}^{i}\right)^{2}+\left((1-w) z_{2}^{i}\right)^{2}\right)^{1 / 2}\right)$, and (c) weighted Tchebycheff $\left(\min _{z^{i} \in \boldsymbol{Z}} \max \left\{w z_{1}^{i},(1-w) z_{2}^{i}\right\}\right)$ functions as in Lokman et al. (2016). We use 10 different weight vectors: $\boldsymbol{w}_{1}=(0.05,0.95)^{T}$, 
Table 1

Average percentage deviations of the preference function values from that of the Decentralized solution ${ }^{\mathrm{a}}$

\begin{tabular}{lllr}
\hline \multirow{2}{*}{ Version } & \multicolumn{2}{l}{ Underlying preference function } & Weighted Tchebycheff \\
\cline { 2 - 4 } & Weighted linear & Weighted Euclidean & -0.8943 \\
\hline$Q C X-u(e r)$ & 0.0486 & -0.0532 & 0.7273 \\
$Q C X-u($ lr $)$ & 0.0110 & -0.0962 & -0.5145 \\
$Q C X-u($ band) & 0.0171 & -0.0251 & -0.9277 \\
$Q C X-u(m n q)$ & 0.0244 & -0.0723 & \\
\hline
\end{tabular}

${ }^{\text {a }}$ Based on 10 instances with different weight values in each cell.

Table 2

Average number of comparisons (average total number of nondominated solutions) with different versions of $Q C X$ - $u^{\mathrm{a}}$

\begin{tabular}{llll}
\hline \multirow{2}{*}{ Version } & \multicolumn{2}{l}{ Underlying preference function } & Weighted Tchebycheff \\
\cline { 2 - 4 } & Weighted linear & Weighted Euclidean & $43.2(308.2)$ \\
\hline$Q C X-u(e r)$ & $31.7(298.1)$ & $46.7(307.5)$ & $32.3(373.4)$ \\
$Q C X-u($ lr $)$ & $19.3(307.8)$ & $26.5(329.6)$ & $64.3(306.5)$ \\
$Q C X-u($ band $)$ & $39.4(264.0)$ & $70.3(285.5)$ & $37.0(305.4)$ \\
$Q C X-u(m n q)$ & $30.0(319.9)$ & $38.0(308.0)$ & \\
\hline
\end{tabular}

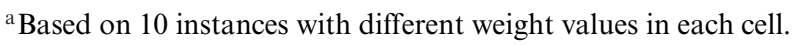

$\boldsymbol{w}_{2}=(0.15,0.85)^{T}, \boldsymbol{w}_{3}=(0.25,0.75)^{T}, \boldsymbol{w}_{4}=(0.35,0.65)^{T}, \boldsymbol{w}_{5}=(0.45,0.55)^{T}, \boldsymbol{w}_{6}=(0.55,0.45)^{T}$, $\boldsymbol{w}_{7}=(0.65,0.35)^{T}, \boldsymbol{w}_{8}=(0.75,0.25)^{T}, \boldsymbol{w}_{9}=(0.85,0.15)^{T}, \boldsymbol{w}_{10}=(0.95,0.05)^{T}$, and generate 10 different problems for each preference function.

Based on our preliminary experiments in $Q C X$ - $u$ (band), we set the predetermined round number, $R$, at which the whole triangle will be searched to 10 . In $Q C X-u(m n q)$, we set the predetermined number used to limit the number of solutions found in the triangles to $M N Q=1$. The average percentage deviations and the average number of comparisons are reported in Tables 1 and 2, respectively.

The results in Table 1 show that in all problems the percentage deviations are very small in each version. Moreover, for underlying nonlinear preference functions, our algorithms performed better than the Decentralized case except for $Q C X-u(l r)$ for an underlying Tchebycheff function for which the average percentage deviation is 0.7273 . Although the Decentralized case has full information about the buyer's preference function, our algorithms may still outperform it for the nonlinear preference functions since all algorithms (including Decentralized) combine different bids linearly. Furthermore, although $Q C X-u$ (lr), $Q C X-u$ (band), and $Q C X-u$ (mnq) only search a portion of the solution space in earlier rounds, they ended up with the most preferred solution of all nondominated solutions generated throughout the problem instance in all instances. $Q C X-u(e r)$, on the other hand, is guaranteed to find the most preferred of all generated nondominated solutions by construction.

In Table 2, we provide the average number of questions asked to the DM and report in parentheses the average total number of nondominated solutions found during the rounds. We should note that the comparisons the DM has to make are cognitively relatively easy since the problem involves only two objectives. The number of pairwise comparisons required by our algorithms is a small percentage 


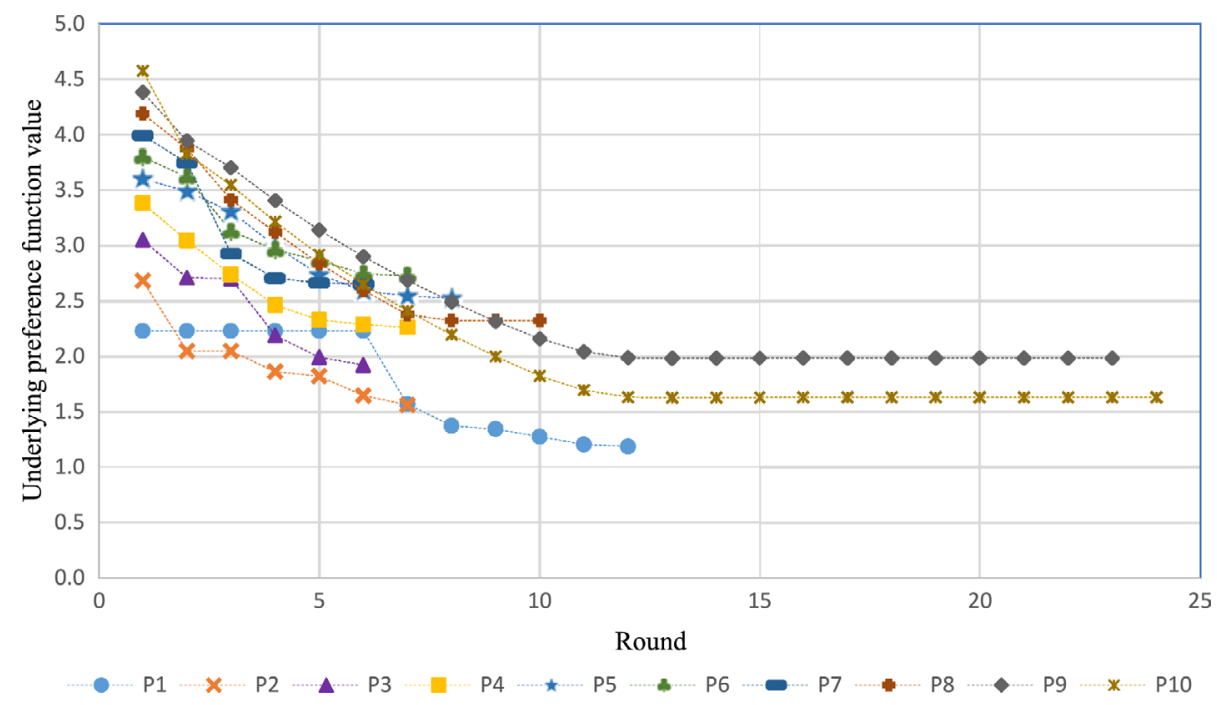

Fig. 5. Underlying preference function values of the solutions of weighted linear preference function case of $Q C X-u(e r)$ at the end of each round for Problems 1-10.

of the number of nondominated solutions that emerge throughout the rounds. The required DM involvement is the least in $Q C X-u(l r)$. In $Q C X-u$ (band), we ask a relatively large number of questions, indicating that the unsupported solutions in the defined band are dense. $Q C X-u$ (er) requires more questions than $Q C X-u(l r)$, but the performances are close in terms of percentage deviations. Although the percentage deviations are very small in each version, considering both the percentage deviations and the number of questions asked to the $\mathrm{DM}$, we can say that $Q C X$ - $u$ (lr) and $Q C X-u(m n q)$ perform better than other versions.

Karakaya and Köksalan (2016) consider a problem setting where sellers update their bids until a predefined termination condition is met, thus they compare the Decentralized solution with the final solution of the corresponding algorithm. On the other hand, in this study we search for a satisfactory solution of the DM. We demonstrate the preference function values of solutions found at the end of each round for the weighted linear case of $Q C X-u(e r)$ and the weighted Tchebycheff case of $Q C X-u(l r)$ in Figs. 5 and 6, respectively. The performances of the algorithms for other cases are similar and we provide the rest of the corresponding results in Figs. A1-A4.

Let us assume that the DM has a stopping rule of $k$ small improvements (say $k=2$ or 3 ). If after the initial improvement a sizeable improvement does not occur within $k$ rounds, he/she stops perceiving the resulting solution as a satisfactory solution. Consider $k=2$. In Problem 10 (P10) for the weighted linear case of $Q C X-u(e r)$, the DM may want to stop at the end of round 15 as there is no improvement for two consecutive rounds after round 13 in the preference function values of the corresponding final solutions. Then, the DM does not need to proceed nine more rounds and make additional judgments. In this example, the DM will end up with the same solution as that obtained at the end of round 24. In the fourth problem of $Q C X-u(l r)$ for the underlying weighted Tchebycheff preference function, suppose that the DM stops at the end of the fourth round since there is no improvement for two consecutive rounds after round 2. There is a loss in this case since the DM would have ended up with a better solution had he/she continued until round 7. In general, 


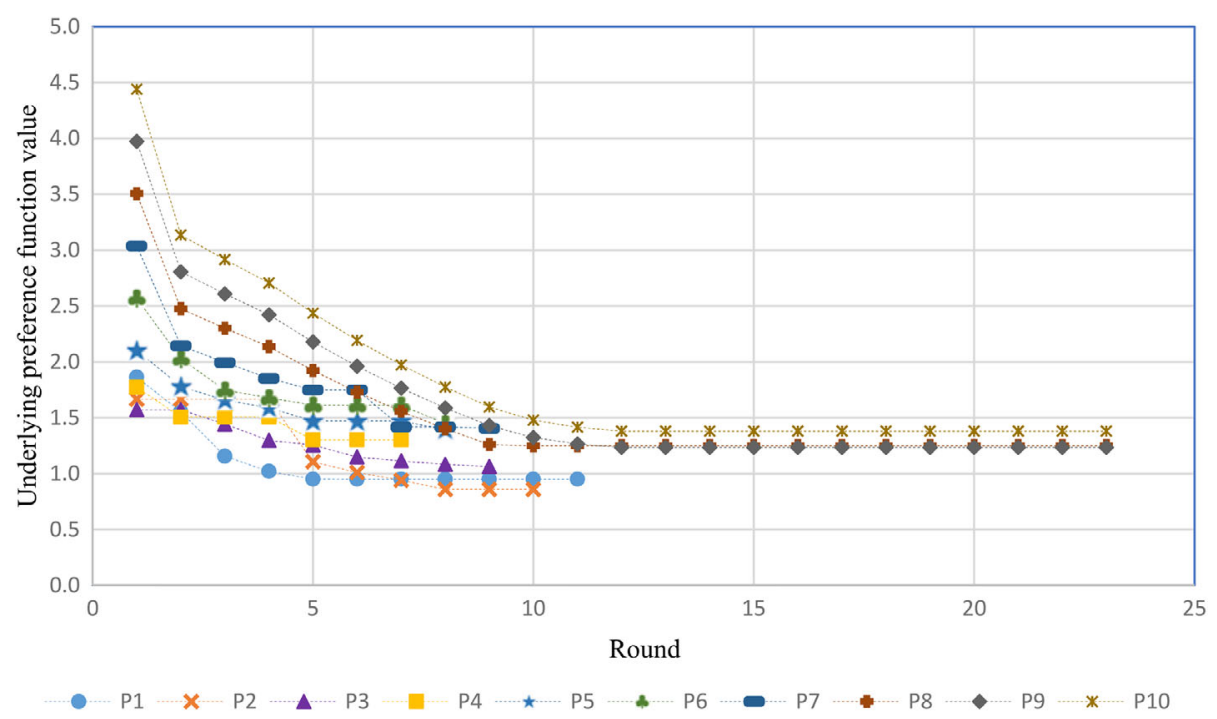

Fig. 6. Underlying preference function values of the solutions of weighted Tchebycheff preference function case of $Q C X-u(l r)$ at the end of each round for Problems 1-10.

the determination of a satisfactory solution is not straightforward unless we have a real DM. For the previous problem, a DM may think that round 4 is too early to stop and he/she may continue even if there is no improvement for two consecutive rounds. Overall, when using a stopping rule of less than a very small threshold improvement (we used $0.05 \%$ in our results) in the preference function value for $k=2$, we observe 4 of 120 cases where the DM would have obtained a somewhat worse solution than the best he/she could have achieved. If we set $k=3$, in all but one of the 120 cases the DM would have obtained the same solution by stopping early as he/she would obtain had he/she continued to the end. In the remaining case, the improvement would have been very small (only $0.008 \%$ ) had the DM continued 10 more rounds.

\section{Conclusions}

In this study, we develop interactive approaches to find a satisfactory solution of a DM having a quasiconvex preference function of two objectives where the set of solutions changes progressively. At each round we first find the most preferred supported solution and then search among unsupported solutions. By keeping the best solution among the ones asked to the DM up to the current round, our algorithm aims to converge to the best solution of the corresponding round.

We develop different versions of the algorithm based on the precision we wish to impose on the preference function value (i.e., the quality) of the obtained solution. The higher precision tends to lead to more preference information required from the DM. Therefore, there is a trade-off between the quality of the solution and the required preference information; the two performance measures we use in evaluating the variations of the algorithm. We test our algorithm on biattribute, multiitem, multi-round auction problems and evaluate their performances. We report their results for 
the case where the DM proceeds until the end of the rounds based on a predetermined termination rule. Our algorithm works well in terms of both measures and finds highly preferred solutions even if the DM decides to terminate after a small number of iterations.

In practice, decision problems typically require considering multiple objectives. However, many approaches in the literature consider a single objective. Adding a second objective enriches such problems substantially as it is then possible to consider cost-related issues with one of the objectives and benefit-related issues with the other objective. Our approaches have been developed for biobjective problems in order to be able to efficiently search the solution spaces under rather general preference structures. Extending our approaches to more than two objectives is a subject of future research.

There have been little research conducted on multi-objective problems for progressively changing solution sets. There could be a variety of structures in which such problems arise. Depending on the structure, the new solution generation could be either straightforward, as in the case of new houses entering the market for sale, or computationally demanding, as in multi-objective combinatorial optimization problems such as the auction problem. We believe that our approaches will attract new interests to optimization problems with progressively changing solution sets.

\section{References}

Chinneck, J.W., 2008. Feasibility and Infeasibility in Optimization: Algorithms and Computational Methods. Springer, New York.

Chun, Y.H., 2015. Multi-attribute sequential decision problems with optimizing and satisficing attributes. European Journal of Operational Research 243, 224-232.

Karakaya, G., Köksalan, M., 2016. An interactive approach for bi-attribute multi-item auctions. Annals of Operations Research 245, 1, 97-119.

Köksalan, M., Karwan M.H., Zionts, S., 1984. An improved method for solving multiple criteria problems involving discrete alternatives. IEEE Transactions on Systems, Man, and Cybernetics 14, 1, 24-34.

Köksalan, M.M., Sagala, P.N., 1995. An approach to and computational results on testing the form of a decision maker's utility function. Journal of Multi-Criteria Decision Analysis 41, 7, 189-202.

Korhonen, P., Moskowitz, H., Salminen, P., Wallenius, J., 1993. Further developments and tests of a progressive algorithm for multiple criteria decision making. Operations Research 6, 41, 1033-1045.

Korhonen, P., Moskowitz, H., Wallenius, J., 1986. A progressive algorithm for modeling and solving multiple criteria decision problems. Operations Research 34, 726-731.

Korhonen, P., Wallenius, J., Zionts, S., 1984. Solving the discrete multiple criteria problem using convex cones. Management Science 30, 1, 1336-1345.

Lokman, B., Köksalan, M., Korhonen, J.P., Wallenius, J., 2016. An interactive algorithm to find the most preferred solution of multi-objective integer programs. Annals of Operations Research 245, 1, 67-95.

Özpeynirci, Ö., Özpeynirci, S., Kaya, A., 2017. An interactive approach for multiple criteria selection problem. Computers \& Operations Research 78, 154-162.

Ramesh, R., Karwan, M.H., Zionts, S., 1990. An interactive method for bicriteria integer programming. IEEE Transactions on Systems, Man, and Cybernetics 20, 2, 395-403.

Ramesh, R., Zionts, S., Karwan, M.H., 1988. Theory of convex cones in multicriteria decision making. Annals of Operations Research 16, 131-148.

Silberberg, E., Suen, W.C., 2001. The Structure of Economics: A Mathematical Analysis (3rd edn). McGraw-Hill, New York.

Simon, H., 1972. Theories of bounded rationality. IEEE Transactions on Systems, Man, and Cybernetics 20, 2, $395-403$.

Zionts, S., 1981. A multiple criteria method for choosing among discrete alternatives. European Journal of Operational Research 7, 2, 143-147. 


\section{Appendix}

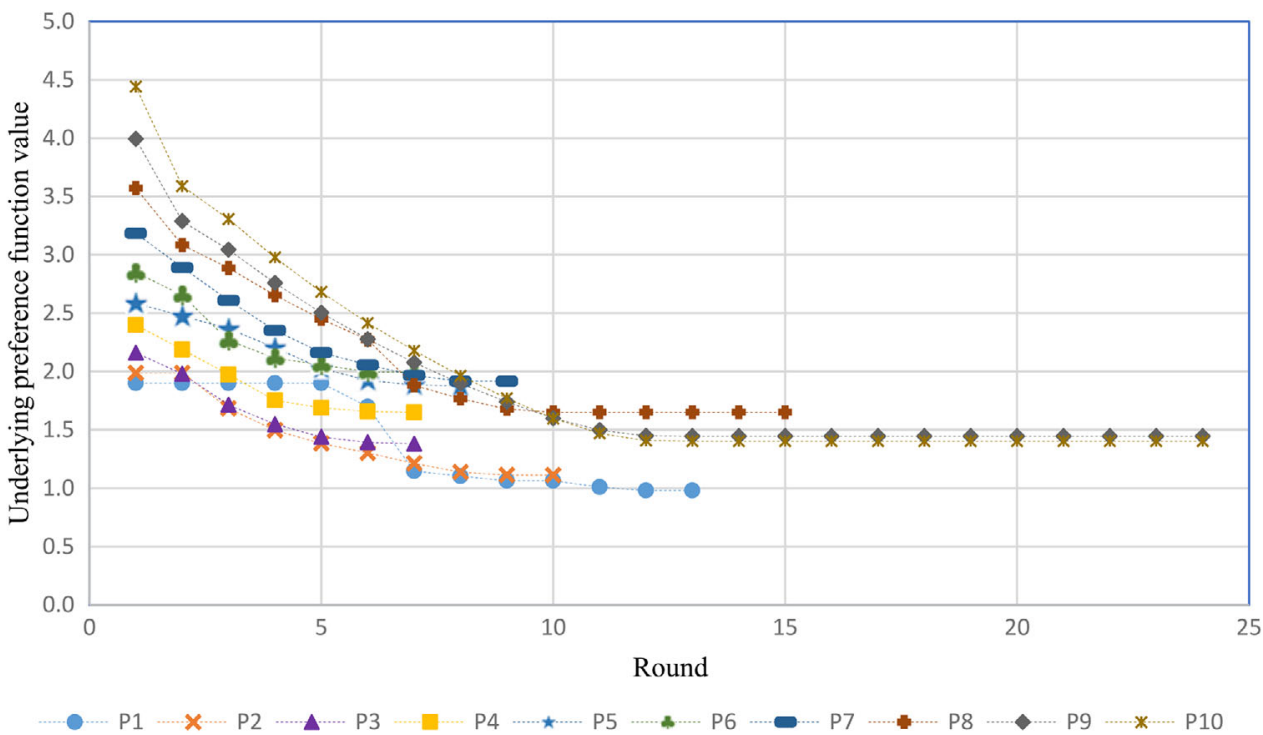

(a) Weighted Euclidean preference function case

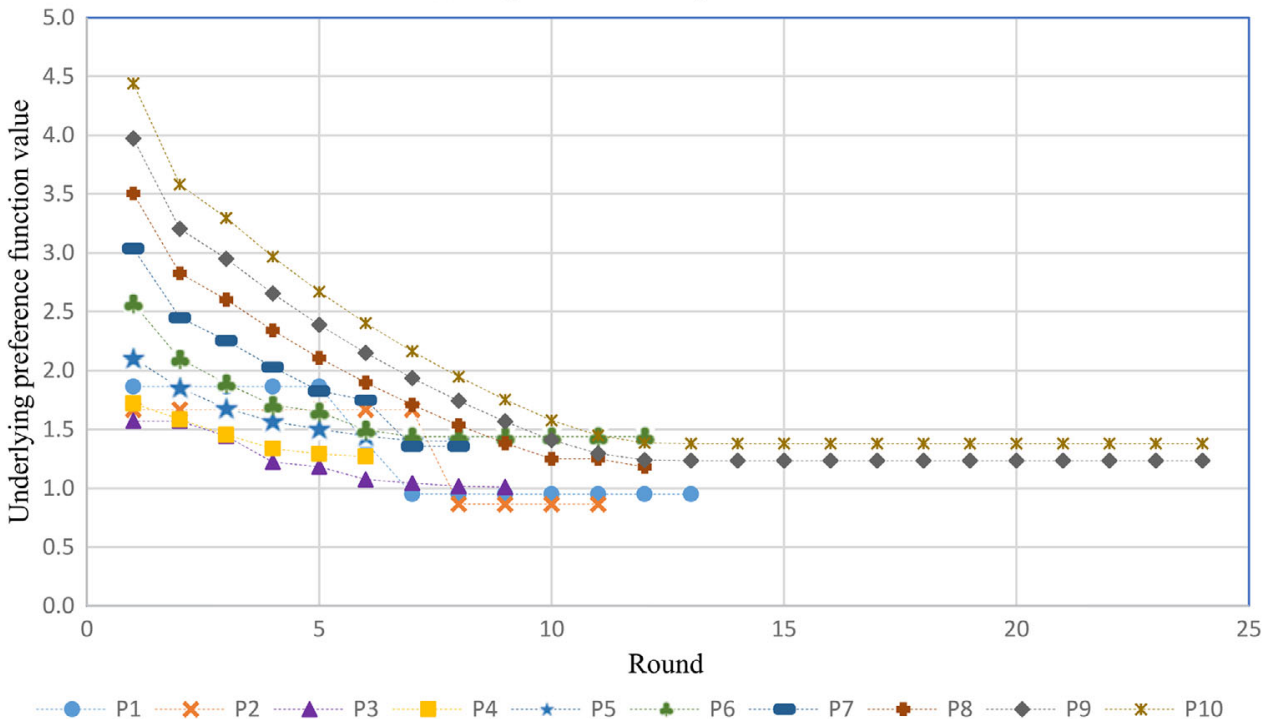

(b) Weighted Tchebycheff preference function case

Fig. A1. Underlying preference function values of the solutions of $Q C X-u(e r)$ at the end of each round for Problems 1-10.

(C) 2018 The Authors.

International Transactions in Operational Research (C) 2018 International Federation of Operational Research Societies 


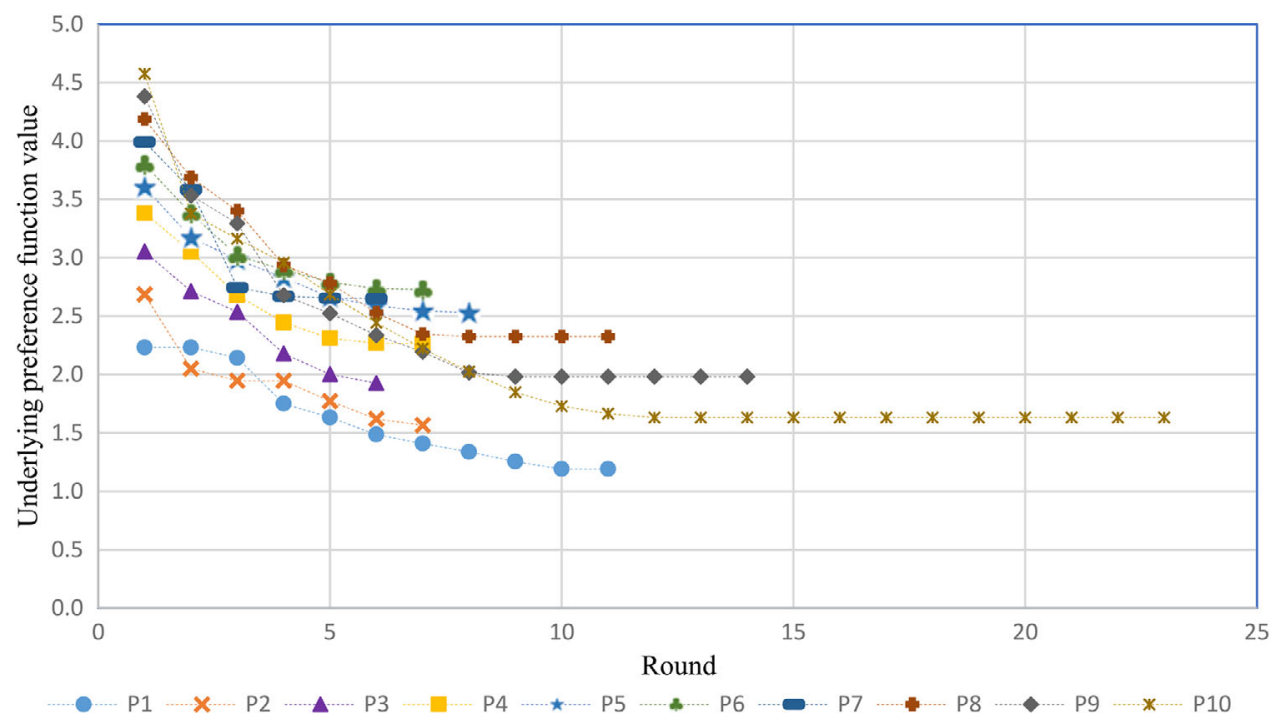

(a) Weighted Linear preference function case

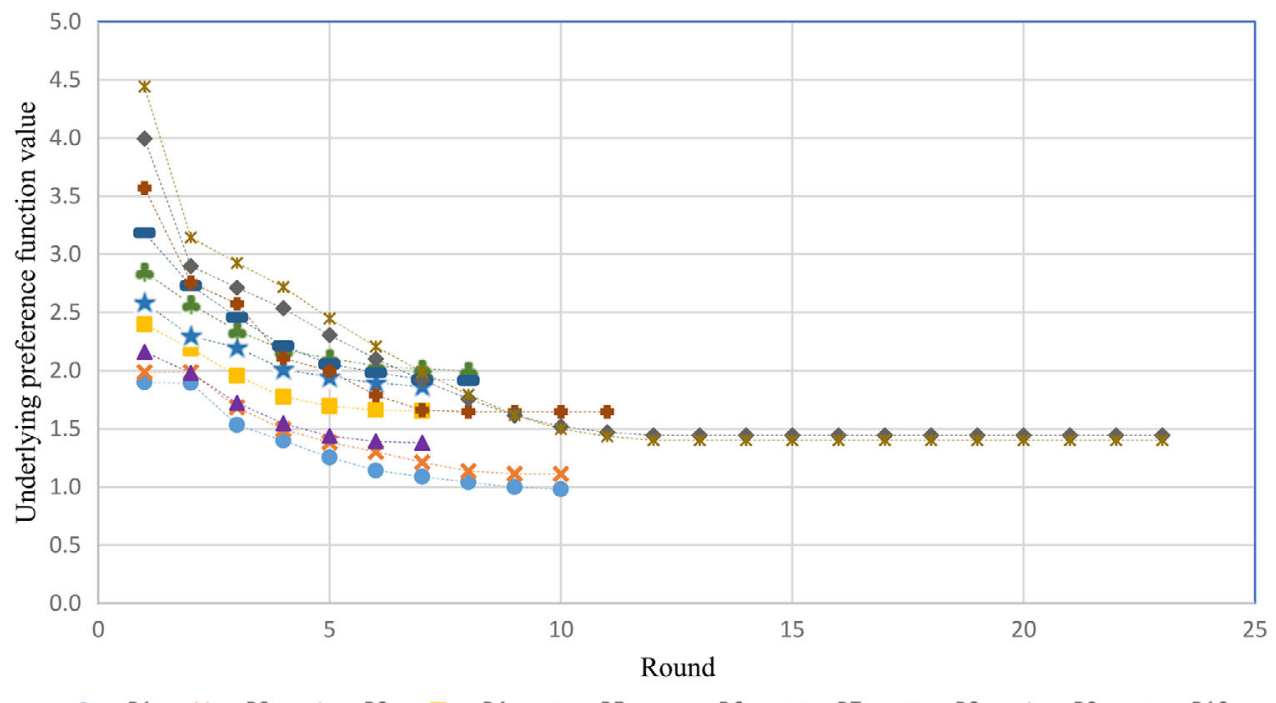

- P1 $\mathrm{P} 1 \mathrm{P} 2 \Delta \mathrm{P} 3 \square \mathrm{P} 4 \star \mathrm{P} 5 * \mathrm{P} 6-\mathrm{P} 7 * \mathrm{P} 8 \bullet \mathrm{P} 9 * \mathrm{P} 10$

(b) Weighted Euclidean preference function case

Fig. A2. Underlying preference function values of the solutions of $Q C X-u(l r)$ at the end of each round for Problems 1-10. 


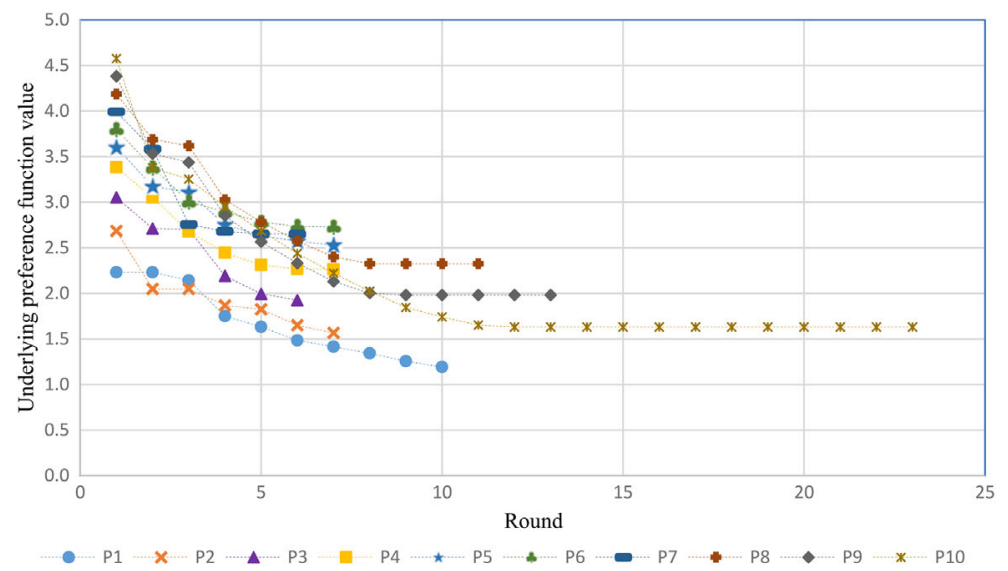

(a) Weighted Linear preference function case

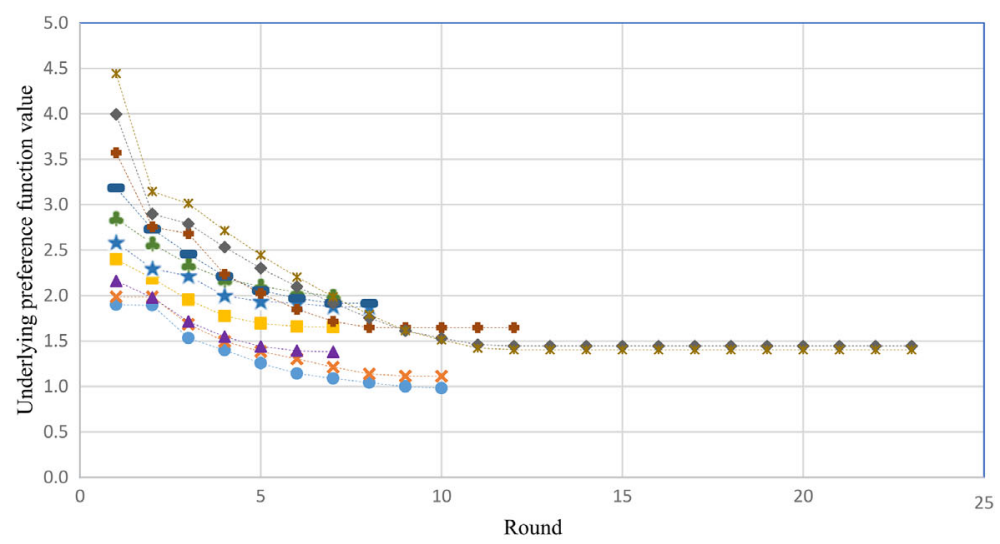

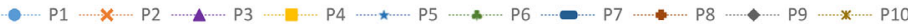

(b) Weighted Euclidean preference function case

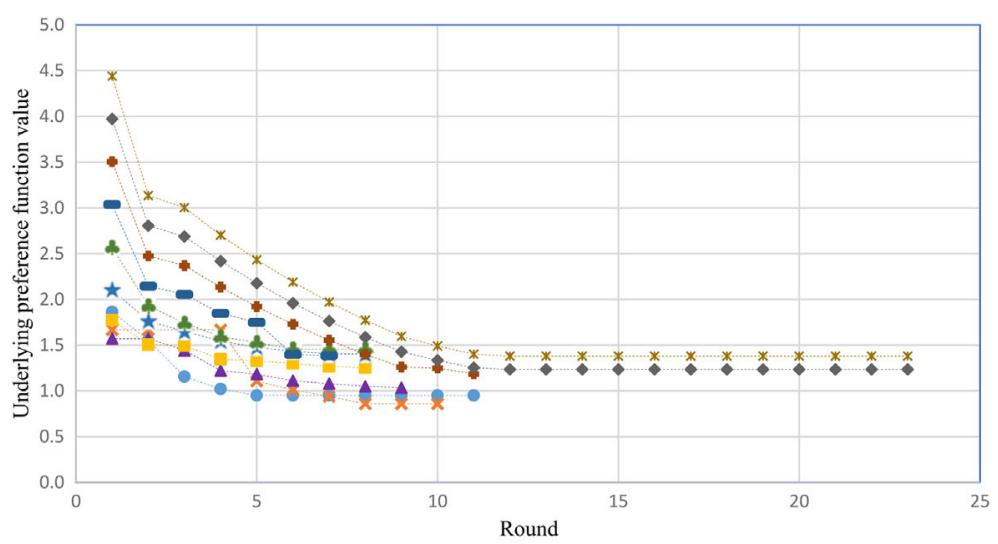

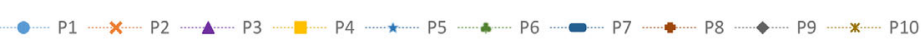

(c) Weighted Tchebycheff preference function case

Fig. A3. Underlying preference function values of the solutions of $Q C X-u$ (band) at the end of each round for Problems 1-10.

(C) 2018 The Authors.

International Transactions in Operational Research (C) 2018 International Federation of Operational Research Societies 


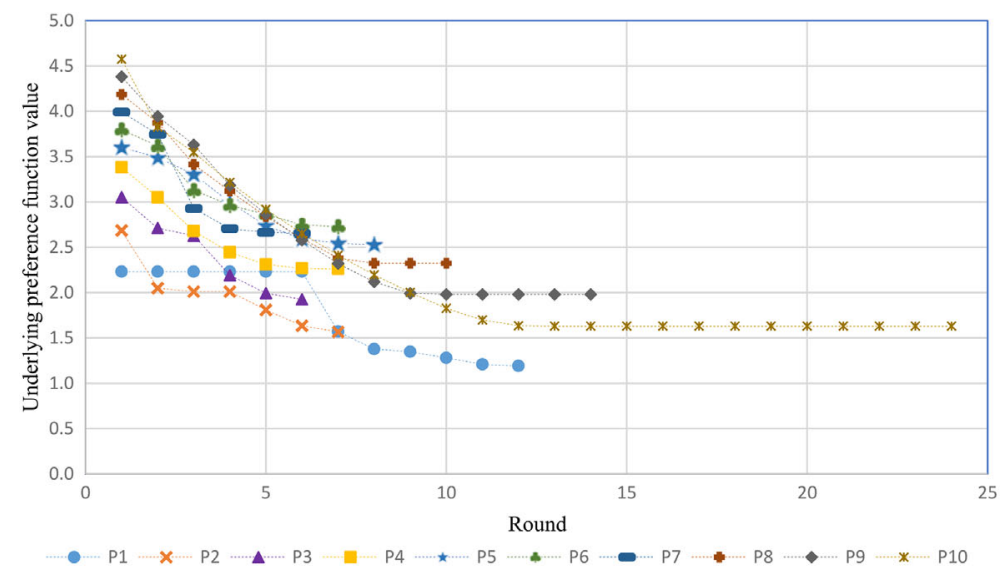

(a) Weighted Linear preference function case
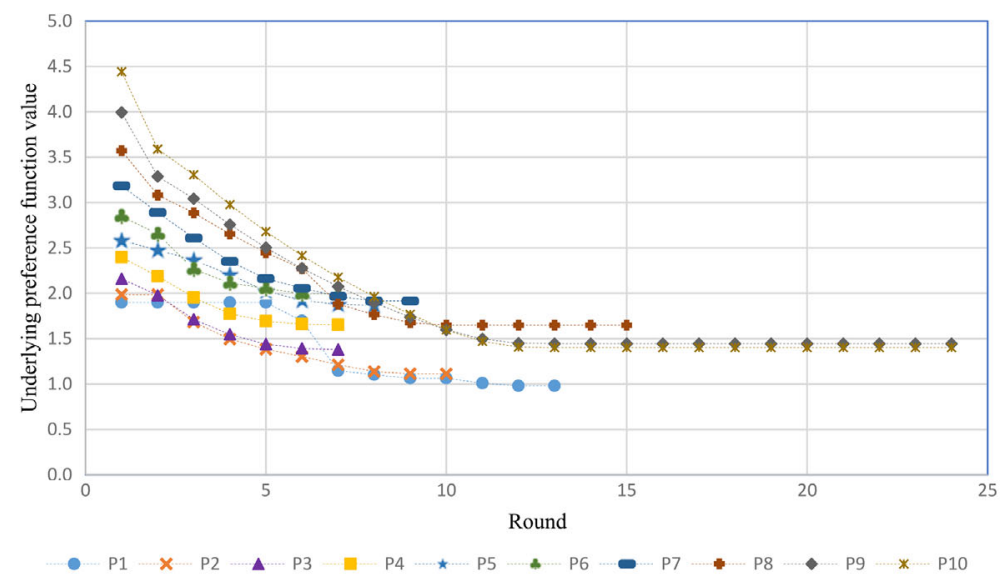

(b) Weighted Euclidean preference function case

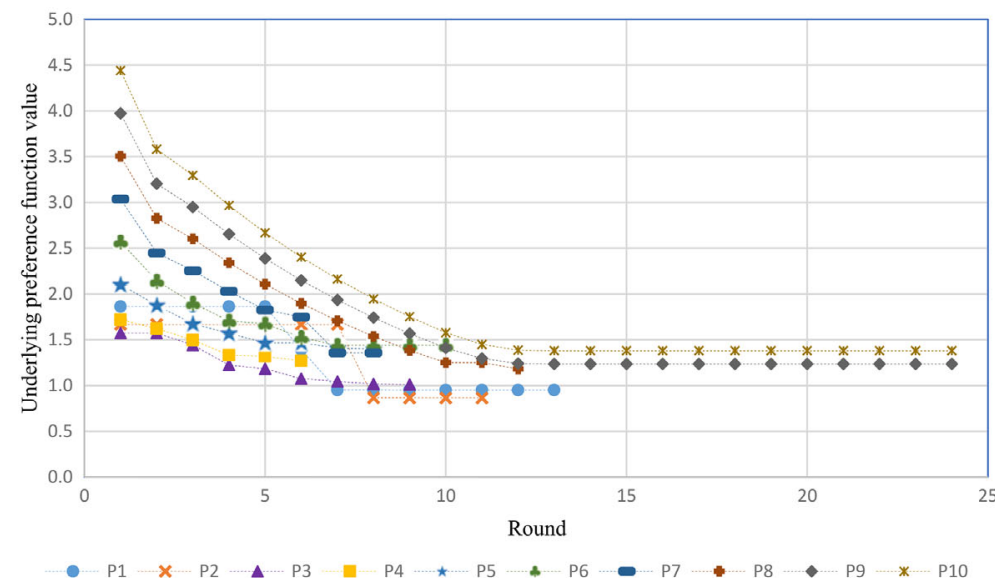

(c) Weighted Tchebycheff preference function case

Fig. A4. Underlying preference function values of the solutions of $Q C X-u(m n q)$ at the end of each round for Problems 1-10. 